\title{
Formation of radial aligned and uniform nematic liquid crystal droplets via drop-on-demand inkjet printing into a partially-wet polymer layer
}

Ellis Parry ${ }^{*}$, Dong-Jin Kim, Alfonso A. Castréjon-Pita*, Steve J. Elston, and Stephen M. Morris ${ }^{*}$ Department of Engineering Science, University of Oxford, Parks Road, Oxford, OX1 3PJ, United Kingdom

*Corresponding author email addresses: ellis.parry@eng.ox.ac.uk, alfonso.castrejonpita@eng.ox.ac.uk, stephen.morris@eng.ox.ac.uk

\begin{abstract}
This paper investigates the drop-on-demand inkjet printing of a nematic liquid crystal (LC) onto a variety of substrates. Achieving both a well-defined droplet boundary and uniformity of the LC director in printed droplets can be challenging when traditional alignment surfaces are employed. Despite the increasing popularity of inkjet printing LCs, the mechanisms that are involved during the deposition process such as drop impact, wetting and spreading have received very little attention, in the way of experiments, as viable routes for promoting alignment of the resultant LC droplets. In this work, radial alignment of the director and uniformity of the droplet boundary are achieved in combination via the use of a partially-wet polymer substrate, which makes use of the forces and flow generated during droplet impact and subsequent wetting process. Our findings could have important consequences for future LC inkjet applications, including the development of smart inks, printable sensors and lasers.
\end{abstract}

Keywords: inkjet printing; nematic liquid crystals; liquid crystal alignment; microfabrication. 


\section{Introduction}

Nematic liquid crystals (LCs) are a form of soft matter that, due to their anisotropic optical properties and sensitivity to an external electric field, have been fundamental in the development of flat-panel display technology[1]. A conventional LC device consists of a thin $(<$ 10 microns) layer of LC sandwiched between two glass substrates that have been specially treated with an alignment layer, which ensures that the director (the average pointing direction of the molecules) aligns with a preferential orientation and/or tilt [2,3]. The deposition of the alignment layer typically involves a number of steps such as the baking of a thin (100s $\mathrm{nm})$ polyimide layer that is subsequently rubbed in order to promote a macroscopic and uniform alignment of the LC molecules parallel to the rubbing direction $[3,4]$. Other techniques that have been adopted to obtain a macroscopic and pre-defined alignment of the LC include the use of photoalignment layers, monolayers of surfactants, such as silane and lecithin, controlled evaporation of $\mathrm{SiO}$ films and flow induced alignment [5-8]. In all cases, achieving the desired alignment requires considerable preparation and treatment of the surfaces before the introduction of the LC material. To reduce the complexity in the fabrication process and to incorporate modern manufacturing techniques, such as inkjet printing, it would be highly desirable if the requirement of alignment layers and the corresponding processes could be relaxed.

In recent years, drop-on-demand (DoD) inkjet printing has proven to be a highly efficient and scalable fabrication process that allows for the accurate control of both the droplet size and volume, as well as the precise position where the fluid can be deposited onto the substrate. An additional benefit is that the technique allows for a variety of 'inks' to be printed simultaneously 
and at high speeds thereby increasing throughput. Furthermore, these inks can be deposited directly onto a range of substrate architectures including highly conformable substrates. Consequently, DoD inkjet printing is now employed to print a plethora of different materials, such as organic light emitting materials, particulate suspensions, conductive polymers and metalloids, for a range of industries and technologies including displays, pharmaceuticals, bioengineering and printed electronics [9-13]. Moreover, many of these applications have become more accessible to inkjet printing as it is now possible to print complex and nonNewtonian fluids such as liquid crystalline materials, which ordinarily possess surface tension and viscous properties that would otherwise make them incompatible with inkjet printing.

In the past six years, both bespoke and commercial inkjet printing systems have been used to deposit LC materials for a range of technological applications. For example, a report by Aliño et al. demonstrated the fabrication of monodisperse nematic LC dispersions by printing the LC onto a pretreated substrate before then rinsing the substrate with a Polyethylenimine solution thereby releasing the droplets into a dispersion [14]. The results presented therein clearly highlight the benefits of incorporating inkjet printing into the preparation process and the ability to prepare LC monodispersions for optical sensor applications. Additionally, Gardiner et al. have demonstrated that it is possible to print dye-doped chiral nematic LCs onto glass substrates to form optically-pumped thin film lasers [15]. Despite the rather non-uniform alignment (as evidenced from the optical polarizing microscope images), it was found that the helical axis was preferentially oriented normal to the glass substrates and consequently each droplet was found to act as a surface emitting micro-laser. In their report, it was shown that printing the chiral nematic LC directly onto a clean, but untreated glass substrate resulted in a uniform sessile droplet with 
diameters of $\sim 300 \mu \mathrm{m}$. However, the alignment of the director within the droplet was found to be non-uniform and consisted of defects when viewed on a polarizing microscope. It was also noted that printing directly on to glass substrates that had been treated with rubbed and baked polyimide layers resulted in considerable wetting of the surface, which made them unsuitable for the purposes of printed laser sources. In order to achieve the standing helix configuration of the chiral nematic phase, the authors found that such an alignment could be obtained by printing onto a $50 \mu \mathrm{m}$-thick wet polymer layer that was coated onto the glass substrate prior to printing.

Given the findings reported in Ref. 15, the purpose of this study is to determine the conditions required to inkjet print a nematic LC onto a Poly(vinyl alcohol) (PVA) polymer layer so as to achieve a spherical and regular droplet perimeter along with a uniform LC director alignment. Both properties result from the precise conditions of the droplet impact and the subsequent interactions between the LC and the substrate surface. In accordance with the results reported in Ref. 15 for a chiral nematic LC, we find that the deposition of the nematic LC onto untreated glass substrates leads to sessile droplets, but with non-uniform alignment of the LC director. When printing a nematic LC on to rubbed polyimide glass substrates, however, droplets are found to consist of defects in the LC director alignment, although we do not observe significant wetting as noted in Ref. 15. In this paper, we alter the drying conditions of the PVA layer to examine the formation of the droplet on dry, wet, and partially-wet polymer layers. It is found that the optimum droplet configuration of the nematic LC is obtained when the polymer layer is neither fully-wet nor fully-dry, but instead some condition in between whereby a uniform sessile droplet is formed with a uniform radial director alignment. 


\section{Experimental}

\subsection{Drop-on-demand printer and image capture}

The experimental set-up that was used in this study was developed in-house and is shown in Fig. 1. The print-head used throughout the study was a MJ-ABP-01-80 dispenser (Microfab Technologies inc.) with an $80 \mu \mathrm{m}$ nozzle diameter. The nematic LC chosen for this study was the nematogen mixture, E7 (Synthon Chemicals $\mathrm{GmbH} \& \mathrm{Co.KG}$ ), which was supplied to the dispenser via a static pressure syringe pump. This nematic LC was chosen as its macroscopic physical properties, such as the refractive indices as well as the elastic and viscosity coefficients, are well-documented. Furthermore, it is liquid crystalline at room temperature and, importantly for printing, the isotropic phase $\left(\mathrm{T}_{\mathrm{c}}=58^{\circ} \mathrm{C}\right)$ is readily accessible with the heating element fitted to our printhead. Two custom-made heating units with a $20 \mathrm{~W}$ heating element (DBK-HPOS, DBK Enclosures) and K-type thermocouple were used to control both the printhead and printing substrate temperatures to within $1^{\circ} \mathrm{C}$ of accuracy.

Drop formation at the nozzle as well as the subsequent deposition onto the glass substrate was captured using a combination of a high-speed camera (Phantom V12.1) and a halogen highintensity white light source (OSL2 3200K, Thorlabs) that were arranged in a shadowgraphy configuration. The alignment of the director of the LC droplet shortly after deposition onto the substrate was analyzed using a CCD camera (DFK 23U274, The Imaging Source) with linear polarizers that were placed before and after the LC sample, with the transmission axes crossed (as shown in Fig. 1). Accurate positioning of the droplet was achieved using a custom-made $x-y$ motorized stage that could be translated in $50 \mu \mathrm{m}$ increments. Finally, timing synchronization, temperature control and printing specifications were achieved using a purpose-built LabVIEW 
program in combination with a computer-controlled DAQ card (NI USB-6351, National Instruments).

\subsection{Printing Conditions}

The formation of repeatable and homogeneous droplets is critical in printing applications and, in the current study, was achieved by careful selection of the printing parameters. The default printing temperature was chosen to be above the clearing temperature, $T_{\mathrm{c}}$ of the LC, and unless stated otherwise the printing temperature was therefore maintained at $60^{\circ} \mathrm{C}$. At this temperature, the bulk viscosity of the LC is reduced from $75 \mathrm{mPa}$.s to approximately $15 \mathrm{mPa} . \mathrm{s}$, which is sufficiently low to reliably deposit using inkjet printing. Additionally, at this temperature, the anisotropic components of the viscosity disappear, leading to increased homogeneity in the droplet properties. A $120 \mathrm{~V}$ square wave pulse was used to generate a single well-defined droplet (via push-mode, DoD) without the formation of satellite droplets, as can be seen in the inset of Fig. 2a. At this voltage, the resulting droplet velocity, which was determined from the analysis of the high-speed images, was found to be $1.6 \pm 0.2 \mathrm{~ms}^{-1}$.

Once the conditions for ejecting the nematic LC had been defined, the next stage was to consider the process of drop impact on the formation of the final printed droplet irrespective of the nature of the substrate. This process can be separated into two separate regimes: the mechanical impact and the subsequent wetting behavior. Initially, there is the transient process of impact between the LC droplet and the substrate, as seen in Fig. 2aii). The time-scale of droplet impact is given by $\frac{d}{u}=50 \mu \mathrm{s}$, where $d$ is the droplet diameter (before impact with the surface) and $u$ is the droplet velocity. During this period a radial shear force and instantaneous fluid flow 
are generated in the LC droplet. It is worth noting that the duration of the impact process is an order of magnitude less than the response time of the LC director in a nematic droplet, which can be estimated by considering the decay time for a simple Fréedericksz transition

$$
t_{\text {decay }}=\frac{\gamma_{1} d^{2}}{\pi^{2} K}
$$

where $\gamma_{1}$ is the rotational viscosity, $d$ is the droplet radius and $K$ is the average elastic constant. Using values for the nematic LC, E7, this results in a decay time of several hundreds of milliseconds. Thus, it is expected that the resultant forces and flow generated during initial droplet impact do not contribute significantly towards the subsequent alignment of the LC director.

During the impact process it is important to ensure that there is no splashing and that the droplet boundary and spherical droplet shape are maintained. Using fluid properties and droplet velocity, the splashing parameter, defined as $\mathrm{K}=\mathrm{We}(\mathrm{Re})^{1 / 2}$ (where $\mathrm{We}$ and $\mathrm{Re}$ are the Weber and Reynolds numbers, respectively), was found to be $\mathrm{K} 4$, which is well below the critical splashing threshold of K>3000 [16]. Therefore, splashing after impact with the surface was not expected to occur, or indeed was observed, for any of the experimental situations presented in this study. After the mechanical effect of droplet impact has subsided, the remaining process corresponds to the wetting of the substrate surface by the LC droplet. Due to the small $\operatorname{Re}(\sim 1)$ and We $(\sim 5)$ numbers involved during this process, the spreading dynamics of the LC droplet post-impact is dominated by the reduction in surface energy and is expected to be within the wetting region, hence no hysteresis of the contact angle is expected or observed [17]. 


\subsection{Preparation of Polymer Substrate}

The Poly(vinyl alcohol) PVA) treated substrates described in this study were prepared as follows. Solutions of $10 \mathrm{wt} \%$ PVA (80\% hydrolyzed, 9,000-10,000MW, Sigma Aldrich) were prepared in deionized water. This specific PVA compound was selected due its transparent optical properties and low viscosity, allowing it to be easily deposited onto glass microscope slides using simple doctor blade-coating processes. The solutions of PVA in deionized water were heated to a temperature of $60^{\circ} \mathrm{C}$ and agitated for $72 \mathrm{hrs}$ to ensure full mixing. Tape was first applied to the microscope slides to form $70 \mu \mathrm{m}$-thick 'walls' at the edges of the substrates and the PVA solution was then drop cast onto the channel that had been formed by the tape and then coated using doctor blading.

\section{Results and Discussion}

To begin with, we consider the printing of a nematic LC onto a rubbed and baked polyimide layer on a glass substrate. Rubbed polyimide is a common alignment surface used in LC devices as it promotes strong uniform planar anchoring parallel to the rubbing direction with an anchoring energy on the order of $10^{-3} \mathrm{Jm}^{-2}[2]$. For this study, an empty glass cell with rubbed polyimide layers (Instec LC2) was carefully split open and one of the glass substrates was then used as the printing substrate. The nematic LC was printed onto the polyimide substrate held at room temperature $\left(\mathrm{T}=22^{\circ} \mathrm{C}\right)$, using the printing conditions described in the Experimental Section. Optical polarizing microscopy images of the LC droplet after deposition onto the rubbed polyimide substrate are presented in Fig. 2bi). In this case, the upper air/LC interface imposes 
homeotropic anchoring conditions on the nematic director within the droplet, whereas the rubbed polyimide layer induces planar anchoring along the rubbing direction, which is indicated by the blue arrow at the top of the microscope images. The alignment imposed by the top and bottom surfaces when combined result in a splay director profile across the droplet. In the microscope image presented in Fig. 2bi), a disclination line can also be seen that results from the topologically incompatible interface between two oppositely oriented splay director alignments, which is illustrated in the diagram in Fig. 2 [18]. The presence of a disclination line was confirmed in all the droplets printed on to the rubbed polyimide glass substrate.

Importantly, due to the anisotropy in the conditions of the substrate surface, the extent over which the LC droplet spreads was often observed to be different along the rubbing direction compared to that perpendicular to the rubbing orientation, resulting in slightly non-spherical droplet boundaries (an example of which is shown in Fig. 2b). This is caused by both differences in the substrate surface profile and, perhaps more importantly, the dependence of the contact angle on the orientation of the nematic molecules at the interface between the LC boundary and the printing substrate $[19,20]$. As a result of the macroscopic rubbing direction, LC droplets deposited on this surface typically possess polarization dependent optical properties due to the planar alignment of the LC molecules.

Following the deposition of the nematic LC on a rubbed polyimide layer, we then examined the resultant droplet formation on a microscope glass slide that had been cleaned by immersing the slide in a solution of $5 \mathrm{wt} \%$ Decon-90 in deionized water for a period of 2 hours followed by sonication in deionized water for 15 minutes. Untreated amorphous glass induces degenerate planar anchoring, whereby the LC molecules align parallel to the glass surface but with no preferential macroscopic orientation [21]. Fig. 2ci) shows the polarized microscopy 
images of the resulting droplet, which exhibits a well-defined droplet boundary but poor uniformity of the LC director. Nematic LCs are classified as flow-aligning, which means that due to the viscous torque imposed on them, the director tends to align parallel to the direction of flow [22]. An important parameter related to the flow of a nematic LC is the Ericksen number [23],

$$
E_{r}=\frac{\eta v L}{K}
$$

where $\eta$ is the flow viscosity, $v$ is the flow velocity, $\mathrm{L}$ is the characteristic length scale and $K$ the elastic coefficient, which is used to quantify alignment generated by fluid-flow of a nematic LC . For $\mathrm{E}_{\mathrm{r}}$ numbers greater than unity, the flow is sufficient to reorient the LC director, which in our case corresponds to flows exceeding $\sim 3.5 \mu \mathrm{ms}^{-1}$. Post-impact, the time taken for the droplet to reach its final equilibrium configuration is approximately $100 \mathrm{~ms}$, corresponding to a fluid flow of $\approx 10 \mu \mathrm{ms}^{-1}$. Combined with the degenerate planar anchoring conditions of the glass substrate, which can accommodate many orientations of the LC director, it is therefore possible that the wetting process can induce a preferential radial orientation. This is confirmed, in part, by the polarized microscopy images in Fig. 2ci, which indicate that the LC director tends to align radially, which is loosely discernable from the orientation of the dark brushes that align with the transmission axes of the polarizers. However, multiple defects in the droplet are clearly visible, which implies that the flow-driven alignment of the wetting process is insufficient to radially reorient the bulk of the $\mathrm{LC}$ director to form a uniform alignment.

We now consider the impact of a PVA layer on the formation of the droplet configuration and director alignment, which was found previously to be beneficial in forming a uniform and sessile droplet of a chiral nematic LC when a wet PVA layer was used as the printing substrate [15]. Here, we consider three separate conditions: fully-dry, fully-wet, and partially wet. Fig. 3a 
illustrates the procedure for preparing the substrates (as described in the Experimental Section) whereas Fig. 3b shows shadowgraphy images of droplet deposition onto a fully-dry PVA layer that was held at a constant temperature of $\mathrm{T}=22^{\circ} \mathrm{C}$. When untreated, a dry PVA film exhibits degenerate planar anchoring with an anchoring strength of the order of $10^{-5} \mathrm{Jm}^{-2}$ [2]. From the images presented in Fig. 3b, the wetting process that was observed was similar to that seen for cleaned, untreated glass substrates with the final droplet configuration being reached in approximately $100 \mathrm{~ms}$, which was accompanied by an increase in the droplet diameter of $\approx 40 \%$ following the initial impact with the surface. The corresponding optical polarizing microscopy images reveal the presence of multiple defects and poor macroscopic uniformity of the LC director. However, the LC droplet boundary was found to be spherical in shape and was consistent across successively-printed droplets.

For the wet PVA layer, which comprises mostly deionized water, microscope glass slides were again prepared following the process shown in Fig. 3a and described in Section 2; however, there was no drying stage and the LC was deposited soon after spreading of the wet PVA layer. Fig. $3 \mathrm{c}$ shows the captured shadowgraphy images of the deposition process onto a fully-wet PVA layer that was $70 \mu \mathrm{m}$-thick as defined by the spacers and held at a constant temperature of $\mathrm{T}=$ $22^{\circ} \mathrm{C}$. During impact, the droplet was seen to penetrate the PVA layer, which was also noted in Ref. 15 for dye-doped chiral nematic LC. In our study it was assumed that the penetration depth had no effect on the final droplet configuration as there was no observable difference seen when printing onto a thinner $35 \mu \mathrm{m}$-thick wet PVA layer, or when printing at a lower droplet velocity. As can be seen in the images, the LC settles above the PVA layer, due the slightly lower density and the thermodynamic requirement to completely wet the PVA surface. Comparing Fig. $3 \mathrm{~b}$ and $3 \mathrm{c}$, after $100 \mathrm{~ms}$, the LC has spread out substantially upon the wet PVA bed. Due to the extent of 
spreading, after $200 \mathrm{~ms}$ it was almost impossible to distinguish between the LC and the PVA solution.

The polarized microscopy images in Fig. 3c show the resultant LC droplets 5 minutes after deposition. Radial planar anchoring within the droplet is evident, indicated by the dark brushes that align with the transmission axes of the polarizers. Although some uniformity in the director alignment is achieved, after 5 minutes the spreading of the droplet had reached diameters of $600 \mu \mathrm{m}$ and the LC continued to wet the PVA surface: these findings represented the complete wetting case irrespective of the printing temperature (as defined by the heater at the printhead) or the substrate temperature. Although the interaction between the LC and wet PVA will not be identical to the interaction with pure water, it will be very similar. Pure water forms a random planar orientation, which is different to degenerate planar anchoring as the LC director is free to reorient due to the fluidity of the water molecules at the surface [24]. This allows for the LC to reorient at the interface without the requirement to desorb from the surface.

In our study, we find that printing into a fully wet PVA layer results in substantial wetting of the PVA and does not lead to the desired droplet configuration. However, depositing the LC onto a wet PVA solution rather than a dry polymer bed did result in a marked improvement in the uniformity of the LC director. A full description of the exact mechanisms responsible for the improvement in the director alignment is outside the scope of this paper, we can make the following remarks. Firstly, the impact of the LC droplet on the PVA bed is cushioned, thus the process imparts less of an impulse force on the LC in the bulk, which could cause less disruption to the alignment of the LC. Additionally, it is evident that the high composition of water during the wetting process contributed significantly to the quality of the planar orientation. During the drying of polymer solutions, water molecules diffuse to the free 
surface before evaporating, forming a thin layer of water at the air interface. It is entirely feasible that the interaction between the LC and the printing substrate is akin to that of pure water, allowing to the LC molecule to reorient under a flow, even if they have already been adsorbed at the anchoring surface.

To combine the favorable droplet boundary seen when printing onto a dry PVA substrate with the uniformity of the LC director apparent when printing onto a wet PVA substrate, a partially-dry PVA layer was used as the printing substrate. The layer was prepared as illustrated in Fig. 4a, with the thickness of the PVA layer being $70 \mu \mathrm{m}$ when wet and the temperature was held constant. The time between spreading and the printing of the LC was used to quantify the 'dryness' of the substrate. It was found that there was a critical dryness of the PVA that promoted both a defined droplet boundary and uniformity of the LC director. This occurred at approximately $75-95 \%$ of the time taken to for the PVA bed to fully dry, corresponding to approximately 60 minutes of drying in an air environment and at room temperature $\left(\mathrm{T}=22^{\circ} \mathrm{C}\right)$. Fig. 4a shows the high-speed images captured during the deposition process. The droplet was found to reach equilibrium in less than $100 \mathrm{~ms}$ with an almost identical degree of wetting to that observed with the fully-dry PVA substrate, reaching a final diameter of $160 \mu \mathrm{m}$. However, in contrast to the dry PVA substrate, there is a uniform radial alignment of the LC director as can be seen in the microscope images in Fig. $4 \mathrm{~b}$ and Fig. $4 \mathrm{c}$. Even though the wetting process is almost identical to the dry polymer and the droplet boundary formed is stable, the alignment is significantly better. This suggests that there is an increased ability of the LC director to reorient at the substrate surface, which may be due to the presence of a thin layer of water at the free surface of the PVA film. 


\section{Conclusions}

For the inkjet printing of LCs, the choice of printing substrate is of critical importance. In addition to conventional inkjet printing criteria, such as reliability of printing and definition of the droplet boundary, the incorporation of LCs as the functional ink introduces the additional condition related to the alignment of the LC director at the surface. It is a property of the printing substrate that governs the uniformity of the LC director in the bulk of the droplet. Conventional methods such as the rubbing of polymer surfaces or partial melting of the polymer often results in a non-spherical droplet boundary or excessive wetting of the droplet. The vast majority of planar aligned surfaces are anisotropic in nature, which consequently leads to anisotropic spreading of the LC droplet. By printing onto a semi-dry (partially-wet) PVA layer it was found that both a circular droplet boundary and uniformity of the LC director could be achieved simultaneously. This work has important implications for future LC inkjet printing applications by highlighting the need for careful consideration of both the LC director orientation required, and the choice of printing substrate required to achieve such orientation. In the case of printing a radial, symmetrical alignment, we have shown how this can be achieved by printing onto a partially-wet PVA layer, but that the use of dry or fully-wet layers as well as rubbed polyimide substrates do not give the desired droplet configuration and alignment of the nematic LC. 


\section{Acknowledgements}

The authors gratefully acknowledge The Royal Society, the John Fell Fund (Oxford), the Engineering and Physical Sciences Research Council (UK) (EP/M50659X/1) and Merck for financial support. We would like to dedicate this manuscript to our co-author, Dong-Jin Kim, who sadly passed away before completion of the manuscript and was instrumental in the development of this research project. Lastly, we would like to acknowledge the kind donation from Dong-Jin Kim's family, in honor of his work and memory, which facilitated the procurement of vital new lab equipment that allowed the results in this manuscript to be obtained. 


\section{References}

[1] J.W.G. Goodby, P.J. Collings, T. Kato, C. Tschierske, H.F. Gleeson, E.P. Raynes, Handbook of Liquid Crystals, Wiley-VCH Verlag GmbH \& Co. KGaA, Weinheim, Germany, 2014. doi:10.1002/9783527671403.

[2] D.-K. Yang, S.-T. Wu, Fundamentals of Liquid Crystal Devices, John Wiley \& Sons, Ltd, Chichester, UK, 2014. doi:10.1002/9781118751992.

[3] B. Jerome, Surface effects and anchoring in liquid crystals, Reports Prog. Phys. 54 (1991) 391-452. doi:10.1088/0034-4885/54/3/002.

[4] J.A. Castellano, Surface Anchoring of Liquid Crystal Molecules on Various Substrates, Mol. Cryst. Liq. Cryst. 94 (1983) 33-41.

doi:10.1080/00268948308084245.

[5] K. Ichimura, Photoalignment of Liquid-Crystal Systems, Chem. Rev. 100 (2000) 1847-1874. doi:10.1021/cr980079e.

[6] F.J. Kahn, G.N. Taylor, H. Schonhorn, Surface-produced alignment of liquid crystals, Proc. IEEE. 61 (1973) 823-828. doi:10.1109/PROC.1973.9171.

[7] O. Yaroshchuk, Y. Reznikov, Photoalignment of liquid crystals: basics and current trends, J. Mater. Chem. 22 (2012) 286-300.

doi:10.1039/C1JM13485J.

[8] Y. Iimura, J.I. Kusano, S. Kobayashi, Y. Aoyagi, T. Sugano, Alignment control of a liquid crystal on a photosensitive polyvinylalcohol film, Jpn. J. Appl. Phys. 32 (1993) L93-L96. doi:10.1143/JJAP.32.L93.

[9] B. Derby, Inkjet printing ceramics: From drops to solid, J. Eur. Ceram. Soc. 31 (2011) 2543-2550. doi:10.1016/j.jeurceramsoc.2011.01.016. 
[10] B. Derby, Inkjet Printing of Functional and Structural Materials: Fluid Property Requirements, Feature Stability, and Resolution, Annu. Rev. Mater. Res. 40 (2010) 395-414. doi:10.1146/annurev-matsci-070909-104502.

[11] S.H. Ko, J. Chung, N. Hotz, K.H. Nam, C.P. Grigoropoulos, Metal nanoparticle direct inkjet printing for low-temperature 3D micro metal structure fabrication, J. Micromechanics Microengineering. 20 (2010) 125010. doi:10.1088/0960-1317/20/12/125010.

[12] R. Daly, T.S. Harrington, G.D. Martin, I.M. Hutchings, Inkjet printing for pharmaceutics - A review of research and manufacturing, Int. J. Pharm. 494 (2015) 554-567. doi:10.1016/J.IJPHARM.2015.03.017.

[13] M. Singh, H.M. Haverinen, P. Dhagat, G.E. Jabbour, Inkjet printing-process and its applications, Adv. Mater. 22 (2010) 673-685. doi:10.1002/adma.200901141.

[14] V.J. Aliño, K.X. Tay, S.A. Khan, K.-L. Yang, Inkjet Printing and Release of Monodisperse Liquid Crystal Droplets from Solid Surfaces, Langmuir. 28 (2012) 14540-14546. doi:10.1021/la3028463.

[15] D.J. Gardiner, W.-K. Hsiao, S.M. Morris, P.J.W. Hands, T.D. Wilkinson, I.M. Hutchings, H.J. Coles, Printed photonic arrays from self-organized chiral nematic liquid crystals, Soft Matter. 8 (2012) 9977. doi:10.1039/c2sm26479j.

[16] C. Josserand, S.T. Thoroddsen, Drop Impact on a Solid Surface, Annu. Rev. Fluid Mech. 48 (2016) 365-391. doi:10.1146/annurev-fluid-122414-034401.

[17] P.G. de Gennes, Wetting: statics and dynamics, Rev. Mod. Phys. 57 (1985) 827-863. doi:10.1103/RevModPhys.57.827.

[18] S. Chandrasekhar, G.S. Ranganath, The structure and energetics of defects in liquid crystals, Adv. Phys. 35 (1986) 507-596.

doi:10.1080/00018738600101941. 
[19] D. Armitage, Alignment of liquid crystals on obliquely evaporated silicon oxide films, J. Appl. Phys. 51 (1980) 2552. doi:10.1063/1.327978.

[20] A.D. Rey, Capillary models for liquid crystal fibers, membranes, films, and drops, Soft Matter. 3 (2007) 1349. doi:10.1039/b704248p.

[21] S. Kumar, J.-H. Kim, Y. Shi, What Aligns Liquid Crystals on Solid Substrates? The Role of Surface Roughness Anisotropy, Phys. Rev. Lett. 94 (2005) 77803. doi:10.1103/PhysRevLett.94.077803.

[22] P.G. de. Gennes, J. Prost, The physics of liquid crystals, Clarendon Press, 1995.

[23] J.L. Ericksen, Anisotropic fluids, Arch. Ration. Mech. Anal. 4 (1959) 231237. doi:10.1007/BF00281389.

[24] R.J. Carlton, C.D. Ma, J.K. Gupta, N.L. Abbott, Influence of Specific Anions on the Orientational Ordering of Thermotropic Liquid Crystals at Aqueous Interfaces, Langmuir. 28 (2012) 12796-12805. doi:10.1021/la3024293. 
Figures and Captions

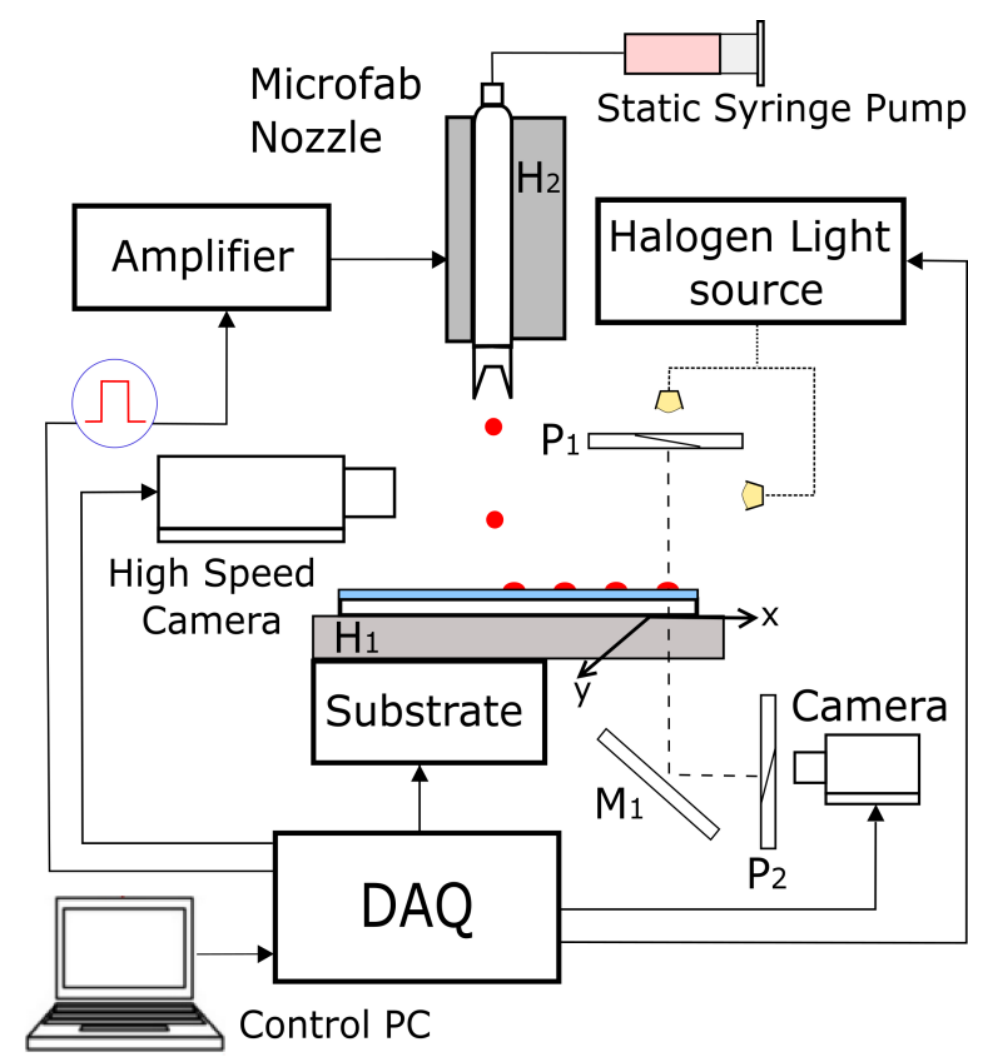

Figure 1. A schematic showing the custom-built experimental apparatus used to print the nematic LC in this study. $\mathrm{P}_{1}$ and $\mathrm{P}_{2}$ are linear polarizers, $\mathrm{H}_{1}$ and $\mathrm{H}_{2}$ are the substrate and nozzle heating elements, respectively, and $\mathrm{M}_{1}$ is a mirror. 


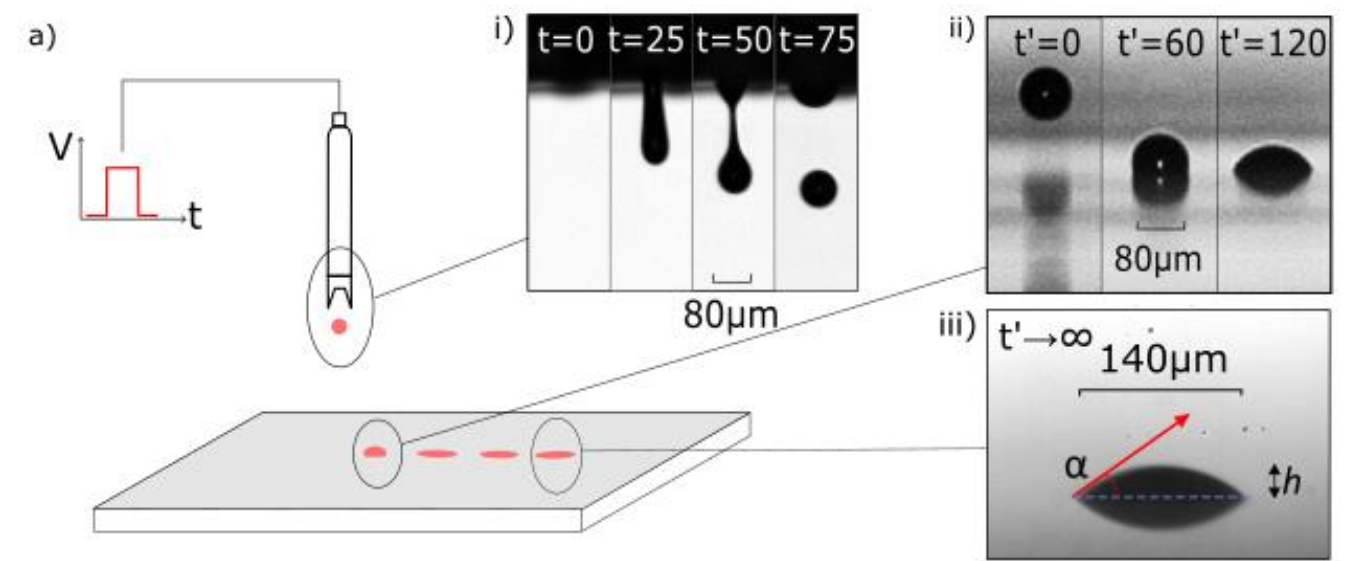

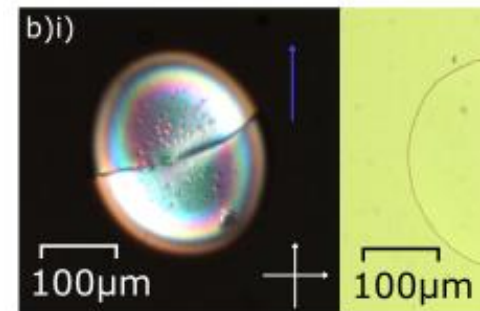

d)

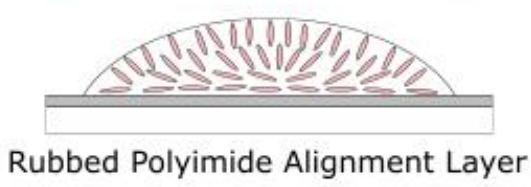

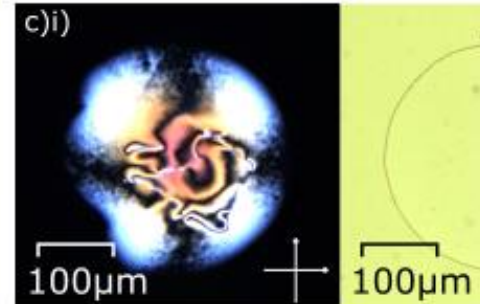

e)

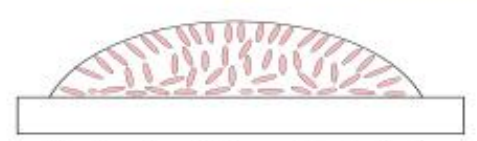

Untreated Glass Substrate

Figure 2. a) Printing of a nematic LC (E7) using an $80 \mu \mathrm{m}$ nozzle at a print temperature of $60^{\circ} \mathrm{C}$ with high-speed images captured at 15,000fps of: i) drop formation at the nozzle with $t$ representing the time from drop emergence $(\mu s)$, ii) drop impact with the printing substrate with t' representing the time of impact with the printing substrate $(\mu \mathrm{s})$, iii) equilibrium droplet profile where $\alpha$ is the contact angle and $h$ is the height of the droplet above the substrate. b)i) Images of a nematic LC droplet printed onto a rubbed polyimide alignment layer when viewed through crossed and uncrossed polarizers: the blue arrow indicates the rubbing direction of the alignment layer and the white arrows represent the transmission axes of the polarisers. c)i) Microscopy images of a nematic LC droplet printed onto a cleaned glass microscope slide viewed through crossed and uncrossed polarizers. d) and e) schematic representation of the LC director 
alignment in a droplet printed onto a rubbed polyimide alignment layer and an untreated glass substrate, respectively.

a)

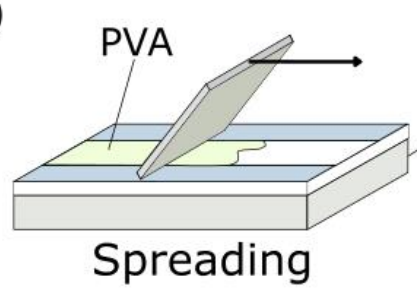

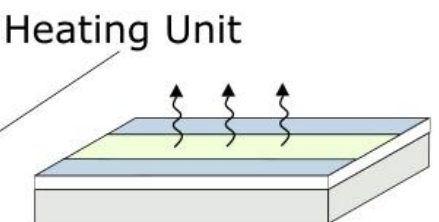

Drying

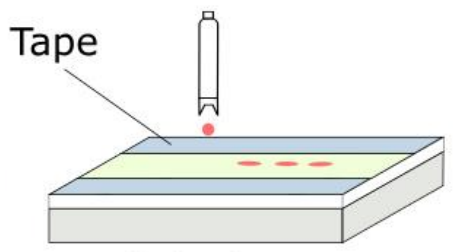

Printing
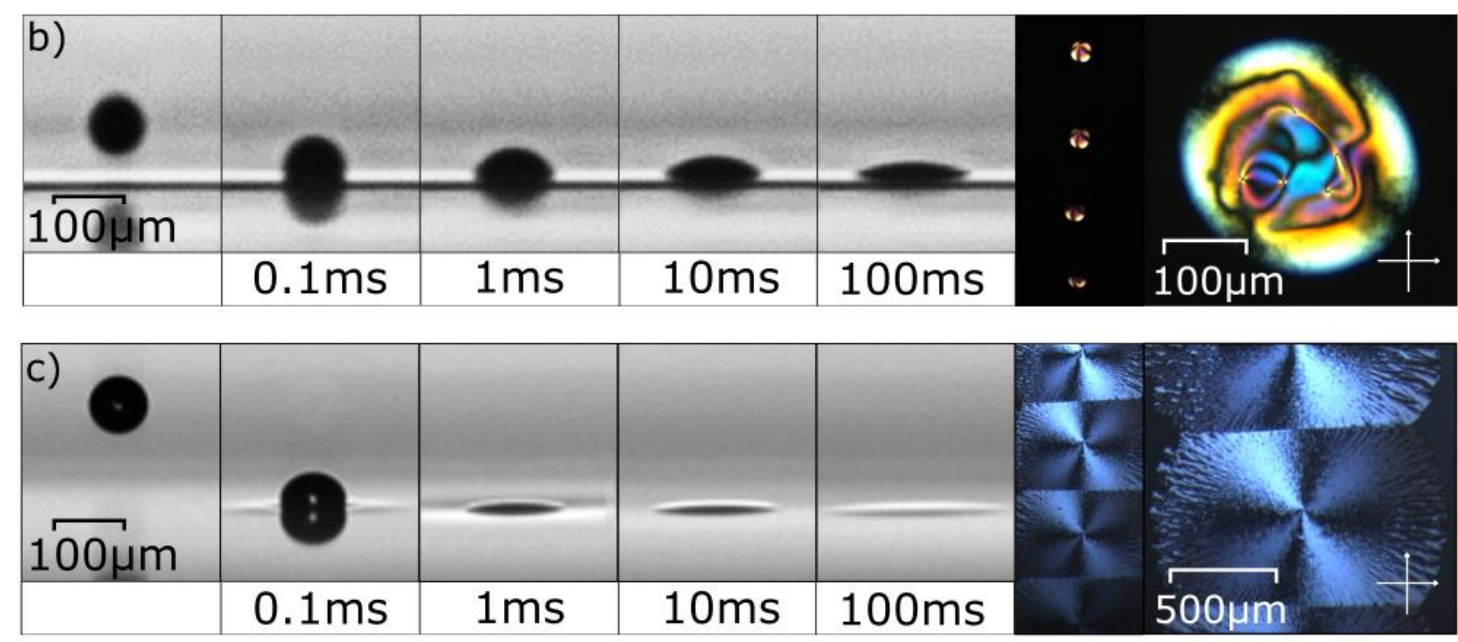

Figure 3 a) Illustration of the procedure used to prepare the PVA-treated substrate. b) Shadowgraphy images captured using the high-speed camera (15,000 fps) showing the impact of the nematic LC, E7, onto a fully-dry PVA layer over a timescale of $100 \mathrm{~ms}$. A polarizing optical microscopy image of the resulting droplet is also shown. c) Shadowgraphy images captured using the high-speed camera (15,000 fps) showing the impact of the nematic LC onto a fully-wet PVA layer over a timescale of $100 \mathrm{~ms}$. A polarizing optical microscopy image of the resulting droplet is also shown under white illumination between crossed polarizers. The white arrows in the crossed configuration in b) and c) represent the orientations of the transmission axes of the polarizers. 

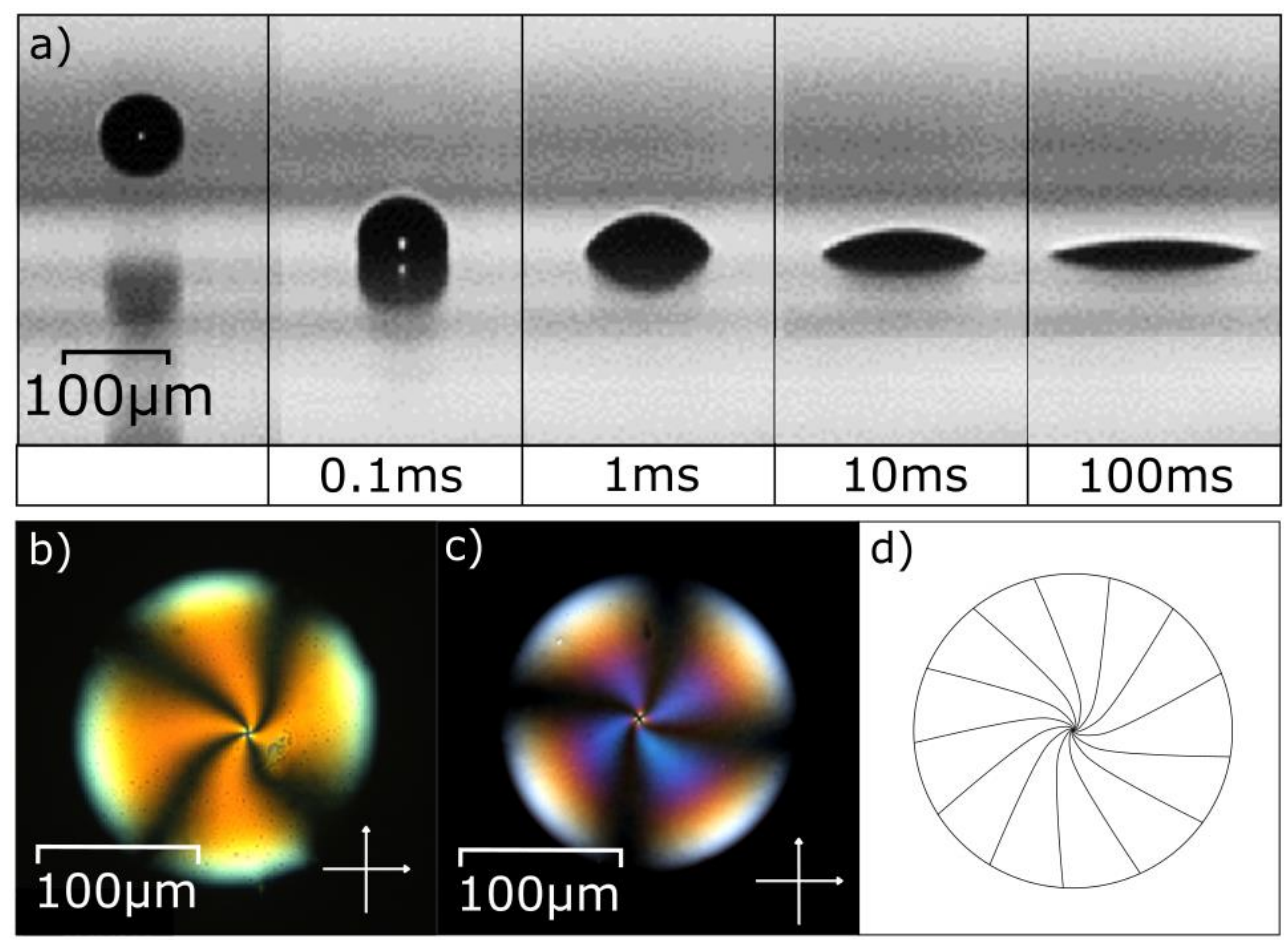

Figure 4. a) Shadowgraphy images captured with the high-speed camera $(15,000 \mathrm{fps})$ of the deposition of the nematic LC onto a partially-dry PVA substrate. b) and c) Polarized microscopy images of a nematic LC droplet printed onto a substrate at $75 \%$ and $90 \%$ dryness, respectively (corresponding to 56 and 63 minutes after spreading). d) An illustration of the resultant radial alignment of the nematic LC director. 